
\title{
$\begin{array}{ll}\text { Research Square } & \begin{array}{l}\text { Preprints are preliminary reports that have not undergone peer review. } \\ \text { They should not be considered conclusive, used to inform clinical practice, } \\ \text { or referenced by the media as validated information. }\end{array}\end{array}$
}

\section{Computed Tomography Findings as Determinants of Local and Systemic Inflammation Biomarkers in Interstitial Lung Diseases: A Retrospective Registry- based Descriptive Study}

\section{David Lang ( $\sim$ david.lang@kepleruniklinikum.at)}

Kepler University Hospital: Kepler Universitatsklinikum GmbH https://orcid.org/0000-0002-3098-1554

\section{Kaveh Akbari}

Kepler Universitätsklinikum GmbH: Kepler Universitatsklinikum GmbH

\section{Andreas Horner}

Kepler University Hospital: Kepler Universitatsklinikum GmbH

Magdalena Hepp

Kepler University Hospital: Kepler Universitatsklinikum GmbH

\section{Bernhard Kaiser}

Kepler University Hospital: Kepler Universitatsklinikum GmbH

\section{Herwig Pieringer}

Diakonissen Hospital Linz

\section{Bernd Lamprecht}

Kepler University Hospital: Kepler Universitatsklinikum GmbH

\section{Research Article}

Keywords: Broncho-alveolar lavage, peripheral blood, C-reactive protein, eosinophil, lymphocyte, idiopathic pulmonary fibrosis, chronic hypersensitivity pneumonitis

Posted Date: February 25th, 2021

DOl: https://doi.org/10.21203/rs.3.rs-246233/v1

License: (c) (i) This work is licensed under a Creative Commons Attribution 4.0 International License. Read Full License

Version of Record: A version of this preprint was published at Lung on March 26th, 2021. See the published version at https://doi.org/10.1007/s00408-021-00434-w. 


\section{Abstract \\ Purpose:}

To evaluate the association of peripheral blood (PBL) and broncho-alveolar lavage (BAL) biomarkers with inflammatory versus fibrotic high-resolution computed tomography (HRCT) findings in interstitial lung diseases (ILD).

\section{Methods:}

HRCT of 127 subsequent ILD-board patients were semi-quantitatively evaluated in a standardized way: Reticulation/honeycombing (RET), traction bronchiectasis (TBR) and emphysema (EMP) were classified as non-inflammatory/fibrotic; consolidations (CON), ground glass opacities (GGO), noduli (NDL) and mosaic attenuation (MOS) as active inflammatory findings. Presence or absence of each HRCT finding was counted in 6 distinct lung regions, resulting scores were graded as minimal ( $0-1$ regions involved), medium (2-4) or extensive (5-6). Associations of routinely assessed PBL/BAL biomarkers with these radiological scores were evaluated using Spearman correlation coefficients and graphical presentation; significance of the graded HRCT scores was tested by applying Kruskal-Wallis tests.

\section{Results:}

Blood neutrophil, lymphocyte and eosinophil fraction, neutrophil-lymphocyte ratio (NLR) and BAL lymphocyte fraction consistently showed opposite correlations for inflammatory versus noninflammatory/fibrotic HRCT finding scores. Blood lymphocyte fraction significantly differed by graded GGO $(p=0.032)$ and CON $(p=0.027)$ extent, eosinophil fraction by TBR $(p=0.006)$ and NLR by CON $(p=$ $0.009)$. C-reactive protein was significantly related to GGO $(p=0.023)$ and CON $(p=0.004)$, BAL lymphocyte fraction to $G G O(p=0.017)$.

\section{Conclusion:}

Blood lymphocyte and eosinophil fraction, NLR, CRP and BAL lymphocyte fraction may aid to differentiate inflammatory from non-inflammatory/fibrotic ILD patterns.

\section{Trial registration:}

This evaluation was based on data from the ILD registry of Kepler University Hospital Linz, as approved by the ethics committee of the federal state of Upper-Austria (EK Nr. I-26-17).

\section{Introduction}


The efficacy of anti-fibrotic drugs in decelerating lung function decline in idiopathic pulmonary fibrosis (IPF) and progressive fibrosing interstitial lung diseases (PFILD) is well established.[1-4] However, patients with ILD susceptible to immunomodulatory therapies can experience an improvement in both radiological imaging and pulmonary function tests,[5-7] while such therapies prove no or even adverse effects in IPF.[8, 9] Importantly, also ILD with an "inflammatory" origin like SSCILD or chronic hypersensitivity pneumonitis (CHP) frequently present with a PFILD phenotype.[10] In systemic sclerosis associated ILD (SSCILD) placebo-controlled trials have provided evidence on the efficacy of immunomodulatory therapies, $[6,11]$ as well as of anti-fibrotic therapy.[12] It is still unclear whether an anti-fibrotic, an immunomodulatory or a combined approach may be most beneficial in such conditions.

In current clinical practice, most biomarker information is derived from high-resolution computed tomography (HRCT) imaging, the cornerstone in the multi-disciplinary diagnosis of ILD. A radiological pattern of usual interstitial pneumonia (UIP) irrespective of the underlying bears a poorer prognosis than possible UIP or nonspecific interstitial pneumonia (NSIP).[13-19] Under certain conditions, an UIP-pattern can be diagnostic for IPF and lead to the initiation of anti-fibrotic therapy without the need of lung biopsy. $[18,19]$ In most ILD cases however, HRCT patterns are not uniform but rather involve several coexisting abnormalities like for example reticulation (RET), ground glass opacities (GGO) and traction bronchiectasis (TBR) in fibrotic NSIP. The relative distribution and extent of such radiological findings may depend on the underlying pathogenetic processes, the course and duration of the disease.[14]

Hypothetically, peripheral blood (PBL) and broncho-alveolar lavage (BAL) biomarkers could help to differentiate ILD cases with an inflammatory from those with a predominantly fibrotic phenotype. Knowledge on their interaction with HRCT findings could aid the development of biomarkers guiding ILD therapy in the future.

\section{Methods}

Based on a retrospective ILD registry cohort, we have evaluated routinely assessed biomarkers from PBL and BAL fluid for their association with a set of visually semi-quantified HRCT finding scores.

This study was performed according to the Strengthening the Reporting of Observational Studies in Epidemiology (STROBE) guidelines for reporting observational studies.[20]

Patient data used for this analysis were retrieved from the ILD registry of Kepler University Hospital Linz, Austria. The registry as well as the present evaluation have been conducted in concordance with the Declaration of Helsinki and were approved and re-assessed on a yearly basis by the ethics committee of the Federal State of Upper Austria (Study number I-26-17). All patients enregistered were subsequently discussed by the monthly local ILD-board after they had undergone a standardized ILD evaluation program including assessment of patient history, physical examination, HRCT imaging, pulmonary function tests and laboratory analyses with standard autoimmune serologies.[19, 21] Patients, in whom ILD board discussion resulted in no diagnosis of an ILD were excluded from this study. 
HRCT images were acquired according to protocols suggested by the relevant guidelines.[18, 19, 22] If clinically feasible, prone imaging was preferred in order to differ opacities in dependent lung areas from true interstitial lung abnormalities.[23]

Blood samples were analyzed with a Sysmex® XN-3000 hematology analyzer (Sysmex Europe GmbH, Norderstedt, Germany) for blood cell counts and a Cobas ${ }^{\circledR} 8000$ modular analyzer (Roche Diagnostics International AG, Rotkreuz, Switzerland) for C-reactive protein (CRP) and lactate dehydrogenase (LDH).

Bronchoalveolar lavage was performed according to the relevant guidelines,[24, 25] when clinically indicated by the treating physician or when requested by the ILD-board. A total of $100 \mathrm{~mL}$ of $0,9 \%$ saline was instilled and retrieved in aliquots of $20 \mathrm{~mL}$ via flexible bronchoscopy under sedoanalgesia. The BAL location was a segmental bronchus of either one of the upper lobes including the lingula or the middle lobe at the discretion of the conducting physician according to the location of most active or extensive disease in HRCT. BAL samples were prepared using $100 \mu \mathrm{L}$ of BAL fluid on a Tharmac ${ }^{\circledR}$ Cellspin I cytocentrifuge (Tharmac $\mathrm{GmbH}$, Wiesbaden, Germany) at 700 rounds per minute for 5 minutes and Wright Giemsa staining. Cell counting was performed manually under 400-fold magnification, cell fractions were given as \% of the total cell count, excluding epithelial cells or erythrocytes.

To allow for statistical analyses of HRCT scans, we have previously devised a semi-quantitative scoring system based on four elementary lesion types: nodular pattern (NDL), reticular abnormalities (interlobular septal and intralobular interstitial thickening and honeycombing - RET), increased lung attenuation (consolidations (CON), ground glass opacities(GGO)) and reduced lung attenuation (emphysema - EMP) findings. Besides, extent of mosaic attenuation (mosaic perfusion, air-trapping - MOS) and traction bronchi(-ol)ectasis (TBR) were assessed. [22, 26, 27]. For quantification, both lungs were separated in an upper-, middle- and lower lung area, as defined by thirds of the largest cranio-caudal diameter in the sagittal reconstructions, leading to six distinct lung areas. For each quantified pattern (RET, TBR, EMP, CON, GGO, NDL, MOS) the individual extent was calculated as the sum of all involved defined lung areas $(0-6)$. The described HRCT scoring process was accomplished during the respective ILD-board session by a specialized ILD radiologist in a non-blinded fashion.

To evaluate the associations between the mentioned inflammation biomarkers with the standardized imaging features, correlation coefficients were calculated for each HRCT finding score and each blood and BAL biomarker. Direction, strength, and significance of these correlations were depicted in colorcoded tables for visual analysis. To test for clinically relevant significance of the associations between HRCT finding scores and the different PBL and BAL biomarkers, groups with no or minimal involvement $(0-1)$, medium (2-5) and extensive involvement (5-6) were compared using the Kruskal-Wallis test. All statistical analyses were performed using R (R: A Language and Environment for Statistical Computing; Version 3.6.0; https://www.R-project.org). For all tests performed, a p-value $<0.05$ was regarded statistically significant.

\section{Results}


We evaluated 127 ILD patients consecutively discussed by the multidisciplinary ILD-board of Kepler University Hospital Linz, Austria between February 2017, and September 2018. Clinical and radiological patient characteristics are shown in Tables 1 and 2. The fraction of patients with "other ILD" $(n=23 ; 18 \%)$ included nine cases of organizing pneumonia (OP), six patients with sarcoidosis, three with respiratorybronchiolitis-ILD, two with drug-associated pneumonitis and one patient each with pulmonary Langerhans-cell histiocytosis, pleuro-parenchymal fibroelastosis and lymphangioleiomyomatosis. Eleven patients $(9 \%)$ were considered "unclassified ILD", either due to patients not willing to undergo further necessary diagnostic steps like lung biopsy or to situations, where further work-up was deemed inappropriate due to age or major comorbidities. Eleven patients (9\%; ten with NSIP, one with unclassifiable ILD) were or had already been diagnosed with autoimmune disorders considered causative or causally related to ILD (rheumatoid arthritis in four patients, autoimmune-hepatitis in two patients, Sjögren's syndrome in two patients and pauci-immune glomerulonephritis, granulomatosis with polyangiitis and SHARP-syndrome in one patient each). 
Table 1

Patient characteristics. Data are given as $\mathrm{n}(\%)$ unless otherwise specified. SD = standard deviation, ILD = interstitial lung disease, IPAF = interstitial pneumonia with autoimmune features, IPF = idiopathic pulmonary fibrosis, $\mathrm{CHP}=$ chronic hypersensitivity pneumonitis, iNSIP = idiopathic non-specific interstitial pneumonia, alLD = autoimmune-associated ILD

\begin{tabular}{|ll|}
\hline Patient Characteristics & \\
\hline Mean age (years, SD) & $65(14)$ \\
\hline Age range (years) & $18-91$ \\
\hline Male Sex (n, \%) & $82(65)$ \\
\hline Reported onset of respiratory symptoms (years, SD) & $4.1(5.8)$ \\
\hline Family history of ILD (n, \%) & $8(6)$ \\
\hline ILD-board diagnosis & $\mathrm{n}(\%)$ \\
\hline IPAF & $26(20)$ \\
\hline IPF & $23(18)$ \\
\hline CHP & $17(13)$ \\
\hline iNSIP & $16(13)$ \\
\hline alLD & $11(9)$ \\
\hline Unclassified ILD & $11(9)$ \\
\hline Other ILD & $23(18)$ \\
\hline Smoking history & $\mathrm{n}(\%)$ \\
\hline Mean pack years (mean, SD) & $19.4(25.2)$ \\
\hline Never smoker & $52(41)$ \\
\hline Former smoker & $50(39)$ \\
\hline Current smoker & $17(13)$ \\
\hline Exclusively passive smoker & $6(5)$ \\
\hline
\end{tabular}


Table 2

Peripheral blood, BAL and HRCT characteristics. Values are given as $\mathrm{n}(\%)$ and mean (SD) or median (range) as specified. $\mathrm{SD}=$ standard deviation, $\mathrm{IQR}=$ interquartile range, $\mathrm{BAL}=$ broncho-alveolar lavage, HRCT = high-resolution computed tomography

\begin{tabular}{|lll|}
\hline Peripheral blood biomarkers & $\mathbf{n}(\%)$ & mean (SD) \\
\hline Leukocyte count (G/L) & $122(96)$ & $8.7(3.4)$ \\
\hline Neutrophil fraction (\%) & $121(95)$ & $70.9(10.9)$ \\
\cline { 2 - 3 } Lymphocyte fraction (\%) & & $20.5(8.7)$ \\
\cline { 1 - 2 } Neutrophil/lymphocyte ratio & & $5(4.9)$ \\
\hline Eosinophil count (\%) & & $0.2(0.3)$ \\
\hline C-reactive protein (mg/dL) & $123(97)$ & $1.6(2.6)$ \\
\hline Lactate dehydrogenase (U/L) & $113(89)$ & $247(78.2)$ \\
\hline BAL biomarkers & $\mathrm{n}(\%)$ & mean (SD) \\
\hline Makrophage fraction (\%) & $66(52)$ & $51.9(29.9)$ \\
\hline Neutrophil fraction (\%) & & $18.5(23.1)$ \\
\hline Lymphocyte fraction (\%) & & $18.5(21.6)$ \\
\hline Eosinophil fraction (\%) & & $3.6(7.9)$ \\
\hline HRCT finding scores & $\mathrm{n}(\%)$ & median (range) \\
\hline Noduli & $40(31)$ & $0(0-6)$ \\
\hline Reticulation/honeycombing & $106(83)$ & $4(0-6)$ \\
\hline Honeycombing & $22(17)$ & $0(0-6)$ \\
\hline Ground glass opacities & $49(39)$ & $0(0-6)$ \\
\hline Consolidations & $44(35)$ & $0(0-6)$ \\
\hline Emphysema & $23(18)$ & $0(0-6)$ \\
\hline Traction bronchiectasis & $100(79)$ & $2(0-6)$ \\
\hline Mosaic attenuation & $32(25)$ & $0(0-6)$ \\
\hline
\end{tabular}

HRCT, PBL and BAL characteristics according to ILD-board diagnoses are shown in Table 3. 
Table 3

HRCT findings, peripheral blood and BAL characteristics according to ILD-board diagnoses. Values are given as median (range) or mean (SD) as specified. SD = standard deviation, HRCT = high-resolution computed tomography, ILD = interstitial lung disease, IPAF = interstitial pneumonia with autoimmune

features, IPF = idiopathic pulmonary fibrosis, $\mathrm{CHP}=$ chronic hypersensitivity pneumonitis, iNSIP = idiopathic non-specific interstitial pneumonia, alLD = autoimmune-associated ILD, RET =

reticulation/honeycombing, $\mathrm{TBR}=$ traction bronchiectasis, $\mathrm{EMP}=$ emphysema, $\mathrm{GGO}=$ ground glass opacities, $\mathrm{CON}$ = consolidations, $\mathrm{NDL}=$ noduli, MOS = mosaic attenuation, HRCT = high-resolution computed tomography, $\mathrm{PBL}=$ peripheral blood, $\mathrm{LEU}=$ absolute leukocyte count, $\mathrm{NEU}=$ relative neutrophil

fraction, LYM = relative lymphocyte fraction, NLR = neutrophil to lymphocyte fraction, EOS = relative eosinophil count, $\mathrm{CRP}=\mathrm{C}$-reactive protein, $\mathrm{LDH}=$ lactate dehydrogenase, $\mathrm{BAL}=$ broncho-alveolar lavage, MAK = relative macrophage fraction

\begin{tabular}{|c|c|c|c|c|c|c|c|}
\hline $\begin{array}{l}\text { HRCT finding scores } \\
\text { (median, range) }\end{array}$ & IPAF & IPF & CHP & iNSIP & alLD & $\begin{array}{l}\text { Unclassified } \\
\text { ILD }\end{array}$ & $\begin{array}{l}\text { Other } \\
\text { ILD }\end{array}$ \\
\hline RET & $\begin{array}{l}5(0- \\
6)\end{array}$ & $\begin{array}{l}6(2- \\
6)\end{array}$ & $6(0-6)$ & $\begin{array}{l}6(2- \\
6)\end{array}$ & $4(2-6)$ & $4(0-6)$ & $\begin{array}{l}0(0- \\
6)\end{array}$ \\
\hline TBR & $\begin{array}{l}2(0- \\
6)\end{array}$ & $\begin{array}{l}4(0- \\
6)\end{array}$ & $4(0-6)$ & $\begin{array}{l}3(0- \\
6)\end{array}$ & $2(0-5)$ & $3(0-6)$ & $\begin{array}{l}1(0- \\
4)\end{array}$ \\
\hline EMP & $\begin{array}{l}0(0- \\
2)\end{array}$ & $\begin{array}{l}0(0- \\
6)\end{array}$ & $0(0-2)$ & $\begin{array}{l}0(0- \\
2)\end{array}$ & $0(0-0)$ & $0(0-0)$ & $\begin{array}{l}0(0- \\
4)\end{array}$ \\
\hline GGO & $\begin{array}{l}0(0- \\
6)\end{array}$ & $\begin{array}{l}0(0- \\
6)\end{array}$ & $2(0-6)$ & $\begin{array}{l}0(0- \\
6)\end{array}$ & $0(0-6)$ & $0(0-6)$ & $\begin{array}{l}0(0- \\
6)\end{array}$ \\
\hline NDL & $\begin{array}{l}0(0- \\
6)\end{array}$ & $\begin{array}{l}0(0- \\
0)\end{array}$ & $2(0-6)$ & $\begin{array}{l}0(0- \\
6)\end{array}$ & $0(0-6)$ & $0(0-6)$ & $\begin{array}{l}1(0- \\
6)\end{array}$ \\
\hline CON & $\begin{array}{l}0.5 \\
(0-6)\end{array}$ & $\begin{array}{l}0(0- \\
4)\end{array}$ & $0(0-2)$ & $\begin{array}{l}0(0- \\
6)\end{array}$ & $0(0-3)$ & $0(0-6)$ & $\begin{array}{l}1(0- \\
6)\end{array}$ \\
\hline MOS & $\begin{array}{l}0(0- \\
6)\end{array}$ & $\begin{array}{l}0(0- \\
2)\end{array}$ & $3(0-6)$ & $\begin{array}{l}0(0- \\
6)\end{array}$ & $0(0-3)$ & $0(0-4)$ & $\begin{array}{l}0(0- \\
6)\end{array}$ \\
\hline $\begin{array}{l}\text { Peripheral blood } \\
\text { biomarkers (mean, } \\
\text { SD) }\end{array}$ & IPAF & IPF & CHP & iNSIP & alLD & $\begin{array}{l}\text { Unclassified } \\
\text { ILD }\end{array}$ & $\begin{array}{l}\text { Other } \\
\text { ILD }\end{array}$ \\
\hline PBL LEU (G/L) & $\begin{array}{l}8.6 \\
(3.0)\end{array}$ & $\begin{array}{l}8.4 \\
(2.5)\end{array}$ & $8.2(2.3)$ & $\begin{array}{l}9.4 \\
(3.3)\end{array}$ & $\begin{array}{l}7.5 \\
(3.7)\end{array}$ & $8.2(3.1)$ & $\begin{array}{l}10.0 \\
(5.0)\end{array}$ \\
\hline PBL NEU (\%) & $\begin{array}{l}74.4 \\
(10.2)\end{array}$ & $\begin{array}{l}66.2 \\
(10.7)\end{array}$ & $\begin{array}{l}70.6 \\
(7.5)\end{array}$ & $\begin{array}{l}70.9 \\
(11.4)\end{array}$ & $\begin{array}{l}73.7 \\
(10.8)\end{array}$ & $69.9(11.0)$ & $\begin{array}{l}70.8 \\
(13.5)\end{array}$ \\
\hline PBL LYM (\%) & $\begin{array}{l}18.2 \\
(8.4)\end{array}$ & $\begin{array}{l}24.9 \\
(8.6)\end{array}$ & $\begin{array}{l}20.3 \\
(6.6)\end{array}$ & $\begin{array}{l}19.6 \\
(8.6)\end{array}$ & $\begin{array}{l}19.3 \\
(8.0)\end{array}$ & $19.9(8.3)$ & $\begin{array}{l}20.5 \\
(10.7)\end{array}$ \\
\hline PBL NLR & $\begin{array}{l}6.2 \\
(5.9)\end{array}$ & $\begin{array}{l}3.4 \\
(2.9)\end{array}$ & $4.0(1.7)$ & $\begin{array}{l}4.8 \\
(3.3)\end{array}$ & $\begin{array}{l}4.8 \\
(2.9)\end{array}$ & $4.7(3.5)$ & $\begin{array}{l}6.4 \\
(8.1)\end{array}$ \\
\hline PBL EOS (\%) & $\begin{array}{l}1.4 \\
(1.5)\end{array}$ & $\begin{array}{l}2.5 \\
(2.3)\end{array}$ & $2.3(1.9)$ & $\begin{array}{l}2.9 \\
(4.0)\end{array}$ & $\begin{array}{l}2.0 \\
(2.5)\end{array}$ & $3.9(4.1)$ & $\begin{array}{l}2.0 \\
(2.5)\end{array}$ \\
\hline
\end{tabular}




\begin{tabular}{|llllllll|}
\hline $\begin{array}{l}\text { HRCT finding scores } \\
\text { (median, range) }\end{array}$ & IPAF & IPF & CHP & iNSIP & alLD & $\begin{array}{l}\text { Unclassified } \\
\text { ILD }\end{array}$ & $\begin{array}{l}\text { Other } \\
\text { ILD }\end{array}$ \\
\hline PBL CRP (mg/dL) & 2.1 & 1.4 & $1.3(1.8)$ & 0.7 & 0.6 & $2.8(4.6)$ & 1.7 \\
& $(3.1)$ & $(2.2)$ & & $(1.0)$ & $(0.5)$ & & $(2.2)$ \\
\hline PBL LDH (U/L) & 243 & 230.8 & 273.4 & 259.6 & 302.7 & $257.7(77.8)$ & 206.0 \\
& $(56.2)$ & $(49.1)$ & $(101.3)$ & $(85.7)$ & $(126.1)$ & & $(51.9)$ \\
\hline BAL biomarkers & IPAF & IPF & CHP & iNSIP & alLD & Unclassified & Other \\
(mean, SD) & & & & & & ILD & ILD \\
\hline BAL MAK (\%) & 44.7 & 60.1 & 30.0 & 81.9 & 61.3 & $34.7(16.3)$ & 48.9 \\
& $(28.2)$ & $(31.9)$ & $(21.0)$ & $(10.2)$ & $(39.0)$ & & $(31.0)$ \\
\hline BAL NEU (\%) & 21.8 & 29.3 & 30.9 & 6.9 & 4.5 & $24.8(23.9)$ & 12.8 \\
& $(23.5)$ & $(30.5)$ & $(34.0)$ & $(7.7)$ & $(4.2)$ & & $(13.4)$ \\
\hline BAL EOS (\%) & 4.1 & 7.6 & 12.0 & 1.5 & 2.0 & $12.0(7.0)$ & 3.8 \\
& $(3.9)$ & $(8.2)$ & $(19.4)$ & $(1.3)$ & $(2.8)$ & & $(1.5)$ \\
\hline BAL LYM (\%) & 24.8 & 6.5 & 24.1 & 10.0 & $18(2-$ & $18.4(9.9)$ & 28.1 \\
& $(27.1)$ & $(6.3)$ & $(24.8)$ & $(8.2)$ & $89)$ & & $(23.1)$ \\
\hline
\end{tabular}

Correlations of peripheral blood and BAL biomarkers with HRCT finding scores are visualized in Fig. 1. Blood neutrophil, lymphocyte and eosinophil fraction, neutrophil-lymphocyte ratio (NLR) and BAL lymphocyte fraction showed consistently opposite correlations for inflammatory versus noninflammatory/fibrotic HRCT finding scores. Significant correlations were seen for PBL lymphocyte fraction and GGO $(r=-0.27, p<0.01)$, PBL NLR and GGO $(r=0.23, p<0.05), P B L$ eosinophil fraction and RET $(r=0.2, p<0.05)$ as well as TBR $(r=0.25, p<0.01)$. Concerning CRP, GGO $(r=0.23, p<0.05)$ and CON $(r=0.21, p<0.05)$ showed significant interactions, while LDH was significantly correlated to RET $(r=0.29$, $p<0.01)$, TBR $(r=0.43, p<0.01), G G O(r=0.24, p<0.05)$ and MOS $(r=0.3, p<0.01)$. Concerning BAL biomarkers, only lymphocyte fraction was significantly correlated with HRCT finding scores, namely with GGO $(r=0.34, p<0.01)$ and CON $(r=0.35, p<0.01)$.

As shown in Tables 4 and 5, the categorized scores for the extent of HRCT findings were used to test the clinically meaningful statistical significance on each peripheral blood or BAL biomarker variable. Blood lymphocyte fraction significantly differed by graded GGO $(p=0.032)$ and CON $(p=0.027)$, blood eosinophil fraction by TBR $(p=0.006)$ and blood NLR by CON $(p=0.009)$ extent. CRP was significantly related with GGO $(p=0.023)$ and CON $(p=0.004)$, while LDH showed significant association with RET ( $p$ $=0.01), \operatorname{TBR}(p<0.001), G G O(p=0.049)$ and MOS $(p=0,027)$. Concerning BAL biomarkers, the only significant interaction again could be shown for BAL lymphocyte fraction and GGO $(p=0.017)$. 
Table 4

Peripheral blood biomarkers according to HRCT finding categories. Data are given as median (range). The $p$-value for statistical significance of differences $(p<0.05)$ between the groups was calculated using the Kruskal-Wallis test. Significant association are shown in bold letters and blue color for positive, red for negative associations. RET = reticulation/honeycombing, TBR = traction bronchiectasis, EMP = emphysema, $\mathrm{GGO}=$ ground glass opacities, $\mathrm{CON}=$ consolidations, $\mathrm{NDL}=$ noduli, $\mathrm{MOS}=$ mosaic attenuation, HRCT = high-resolution computed tomography, LEU = absolute blood leukocyte count, NEU = relative blood neutrophil fraction, LYM = relative blood lymphocyte fraction, NLR = neutrophil to lymphocyte fraction, EOS = relative blood eosinophil count, CRP = C-reactive protein, $\mathrm{LDH}=$ lactate dehydrogenase

\begin{tabular}{|c|c|c|c|c|c|c|c|}
\hline & Median in HRCT & core groups (range & & p & $\mathrm{n}$ & & \\
\hline LEU (G/L) & $0-1$ & $2-4$ & $5-6$ & & $\begin{array}{l}0- \\
1\end{array}$ & $\begin{array}{l}2- \\
4\end{array}$ & $\begin{array}{l}5- \\
6\end{array}$ \\
\hline RET & $7.3(4.8-15.9)$ & $7.5(3.8-25.5)$ & $8.5(4.5-16.1)$ & 0.617 & 22 & 46 & 54 \\
\hline TBR & $7.8(4.6-25.5)$ & $7.5(3.8-19.1)$ & $8.5(4.8-14.6)$ & 0.537 & 30 & 66 & 26 \\
\hline EMP & $7.8(3.8-19.1)$ & $8.6(4.6-25.5)$ & $7.2(4.8-14.6)$ & 0.916 & 100 & 19 & 3 \\
\hline GGO & $7.6(3.8-16.1)$ & $7.9(4.2-14.6)$ & $8.3(4.8-25.5)$ & 0.449 & 78 & 24 & 20 \\
\hline CON & $7.8(3.8-25.5)$ & $7.4(4.5-19.1)$ & $9.7(4.8-14.6)$ & 0.811 & 93 & 21 & 8 \\
\hline NDL & $7.6(3.8-19.1)$ & $8.3(4.2-25.5)$ & $9.6(5.6-15.4)$ & 0.117 & 85 & 24 & 13 \\
\hline MOS & $7.7(3.8-25.5)$ & $8.4(4.2-13.3)$ & $7.6(4.8-18.1)$ & 0.839 & 96 & 18 & 8 \\
\hline NEU (\%) & $0-1$ & $2-4$ & $5-6$ & p & $\begin{array}{l}0- \\
1\end{array}$ & $\begin{array}{l}2- \\
4\end{array}$ & $\begin{array}{l}5- \\
6\end{array}$ \\
\hline RET & $\begin{array}{l}74.5(54.4- \\
91.7)\end{array}$ & $\begin{array}{l}72.4(46.2- \\
96.6)\end{array}$ & $\begin{array}{l}68.8(47.6- \\
88.3)\end{array}$ & 0.362 & 22 & 54 & 54 \\
\hline TBR & $\begin{array}{l}74.3(46.2- \\
94.9)\end{array}$ & $\begin{array}{l}69.0(46.2- \\
94.9)\end{array}$ & $\begin{array}{l}73.1(47.6- \\
86.3)\end{array}$ & 0.130 & 30 & 65 & 26 \\
\hline EMP & $\begin{array}{l}71.3(51.4- \\
94.9)\end{array}$ & $\begin{array}{l}69.6(46.2- \\
96.6)\end{array}$ & $\begin{array}{l}69.8(47.6- \\
86.3)\end{array}$ & 0.967 & 99 & 19 & 3 \\
\hline GGO & $\begin{array}{l}69.7(46.2- \\
91.7)\end{array}$ & $\begin{array}{l}71.9(51.9- \\
89.6)\end{array}$ & $\begin{array}{l}74.8(47.6- \\
96.6)\end{array}$ & 0.289 & 78 & 24 & 19 \\
\hline CON & $\begin{array}{l}69.8(46.2- \\
96.6)\end{array}$ & $\begin{array}{l}71.5(51.4- \\
86.3)\end{array}$ & $\begin{array}{l}79.5(58.2- \\
89.6)\end{array}$ & 0.077 & 93 & 20 & 8 \\
\hline NDL & $\begin{array}{l}69.5(46.2- \\
89.6)\end{array}$ & $\begin{array}{l}72.8(54.4- \\
96.6)\end{array}$ & $\begin{array}{l}71.4(51.9- \\
91.7)\end{array}$ & 0.217 & 84 & 24 & 13 \\
\hline MOS & $\begin{array}{l}69.6(46.2- \\
96.6)\end{array}$ & $\begin{array}{l}72.6(51.4- \\
90.2)\end{array}$ & $79.3(61.0-94.9)$ & 0.108 & 95 & 18 & 8 \\
\hline LYM (\%) & $0-1$ & $2-4$ & $5-6$ & p & $\begin{array}{l}0- \\
1\end{array}$ & $\begin{array}{l}2- \\
4\end{array}$ & $\begin{array}{l}5- \\
6\end{array}$ \\
\hline RET & $19.3(4.6-37.4)$ & $19.1(2.6-41.9)$ & $22.3(4.6-38.2)$ & 0.366 & 22 & 45 & 54 \\
\hline
\end{tabular}




\begin{tabular}{|c|c|c|c|c|c|c|c|}
\hline \multicolumn{4}{|c|}{ Median in HRCT score groups (range) } & \multirow[t]{2}{*}{$\mathbf{p}$} & \multicolumn{3}{|l|}{$\mathbf{n}$} \\
\hline LEU (G/L) & $0-1$ & $2-4$ & $5-6$ & & $\begin{array}{l}0- \\
1\end{array}$ & $\begin{array}{l}2- \\
4\end{array}$ & $\begin{array}{l}5- \\
6\end{array}$ \\
\hline TBR & $18.6(2.6-37.4)$ & $21.4(3.4-41.9)$ & $19.3(5.9-38.2)$ & 0.337 & 30 & 65 & 26 \\
\hline EMP & $20.0(3.4-38.0)$ & $20.6(2.6-41.9)$ & $23.2(5.9-38.2)$ & 0.915 & 99 & 19 & 3 \\
\hline GGO & $22.7(4.6-41.9)$ & $16.9(7.8-41.5)$ & $17.9(2.6-38.2)$ & 0.032 & 78 & 24 & 19 \\
\hline CON & $21.0(2.6-41.9)$ & $19.7(5.9-34.2)$ & $13.0(7.8-21.5)$ & 0.027 & 93 & 20 & 8 \\
\hline NDL & $21.1(4.6-41.9)$ & $18.4(2.6-37.4)$ & $21.8(4.6-41.5)$ & 0.150 & 84 & 24 & 13 \\
\hline MOS & $20.9(2.6-41.9)$ & $18.2(6.6-34.8)$ & $15.6(3.4-24.4)$ & 0.154 & 95 & 18 & 8 \\
\hline NLR & $0-1$ & $2-4$ & $5-6$ & $\mathrm{p}$ & $\begin{array}{l}0- \\
1\end{array}$ & $\begin{array}{l}2- \\
4\end{array}$ & $\begin{array}{l}5- \\
6\end{array}$ \\
\hline RET & $4.2(1.5-19.9)$ & $3.8(1.1-37.1)$ & $3.1(1.2-19.3)$ & 0.311 & 22 & 45 & 54 \\
\hline TBR & $4.1(1.5-37.1)$ & $3.2(1.1-27.8)$ & $3.9(1.2-14.7)$ & 0.160 & 30 & 65 & 26 \\
\hline EMP & $3.7(1.4-27.8)$ & $3.5(1.1-37.1)$ & $3.0(1.2-14.7)$ & 0.941 & 99 & 19 & 3 \\
\hline GGO & $3.2(1.1-19.9)$ & $4.2(1.3-11.4)$ & $4.2(1.2-37.1)$ & 0.095 & 78 & 24 & 19 \\
\hline CON & $3.2(1.1-37.1)$ & $3.7(1.5-14.7)$ & $6.4(5.1-11.4)$ & 0.009 & 93 & 20 & 8 \\
\hline NDL & $3.5(1.1-19.3)$ & $4.0(1.5-37.1)$ & $3.2(1.3-19.9)$ & 0.172 & 84 & 24 & 13 \\
\hline MOS & $3.4(1.1-37.1)$ & $4.1(1.5-13.3)$ & $5.1(2.5-27.8)$ & 0.156 & 95 & 18 & 8 \\
\hline EOS (\%) & $0-1$ & $2-4$ & $5-6$ & p & $\begin{array}{l}0- \\
1\end{array}$ & $\begin{array}{l}2- \\
4\end{array}$ & $\begin{array}{l}5- \\
6\end{array}$ \\
\hline RET & $1.0(0.0-5.1)$ & $1.4(0.0-13.8)$ & $1.8(0.1-14.7)$ & 0.092 & 21 & 45 & 52 \\
\hline TBR & $0.9(0.0-4.4)$ & $1.7(0.0-13.8)$ & $1.9(0.1-14.7)$ & 0.006 & 30 & 64 & 24 \\
\hline EMP & $1.5(0.0-14.7)$ & $1.5(0.1-13.8)$ & $1.8(0.1-6.5)$ & 0.909 & 96 & 19 & 3 \\
\hline GGO & $1.5(0.0-9.5)$ & $1.5(0.0-14.7)$ & $1.4(0.0-11.2)$ & 0.973 & 75 & 24 & 19 \\
\hline CON & $1.5(0.0-13.8)$ & $1.7(0.1-14.7)$ & $0.6(0.1-2.5)$ & 0.168 & 91 & 19 & 8 \\
\hline NDL & $1.5(0.0-14.7)$ & $1.6(0.0-13.8)$ & $1.1(0.0-11.2)$ & 0.753 & 82 & 23 & 13 \\
\hline MOS & $1.5(0.0-14.7)$ & $1.8(0.0-8.4)$ & $0.3(0.0-8.6)$ & 0.148 & 93 & 17 & 8 \\
\hline $\begin{array}{l}\text { CRP } \\
(\mathrm{mg} / \mathrm{dL})\end{array}$ & $0-1$ & $2-4$ & $5-6$ & p & $\begin{array}{l}0- \\
1\end{array}$ & $\begin{array}{l}2- \\
4\end{array}$ & $\begin{array}{l}5- \\
6\end{array}$ \\
\hline RET & $0.5(0.1-7.1)$ & $0.4(0.1-8.4)$ & $0.9(0.1-15.9)$ & 0.257 & 22 & 45 & 56 \\
\hline TBR & $0.4(0.1-7.1)$ & $0.7(0.1-12.3)$ & $0.3(0.1-15.9)$ & 0.332 & 30 & 67 & 26 \\
\hline
\end{tabular}




\begin{tabular}{|c|c|c|c|c|c|c|c|}
\hline \multirow[b]{2}{*}{ LEU (G/L) } & \multicolumn{3}{|c|}{ Median in HRCT score groups (range) } & \multirow[t]{2}{*}{$\mathrm{p}$} & \multicolumn{3}{|l|}{$\mathrm{n}$} \\
\hline & $0-1$ & $2-4$ & $5-6$ & & $\begin{array}{l}0- \\
1\end{array}$ & $\begin{array}{l}2- \\
4\end{array}$ & $\begin{array}{l}5- \\
6\end{array}$ \\
\hline EMP & $0.5(0.1-15.9)$ & $0.6(0.1-12.3)$ & $0.3(0.1-5.0)$ & 0.917 & 101 & 19 & 3 \\
\hline GGO & $0.4(0.1-12.3)$ & $1.0(0.1-15.9)$ & $0.9(0.1-8.4)$ & 0.023 & 80 & 23 & 20 \\
\hline CON & $0.5(0.1-12.3)$ & $0.4(0.1-15.9)$ & $4.1(0.6-8.4)$ & 0.004 & 93 & 22 & 8 \\
\hline NDL & $0.4(0.1-15.9)$ & $0.9(0.1-6.8)$ & $0.7(0.1-7.1)$ & 0.221 & 87 & 23 & 13 \\
\hline MOS & $0.5(0.1-15.9)$ & $0.7(0.1-3.8)$ & $0.9(0.2-6.0)$ & 0.393 & 98 & 17 & 8 \\
\hline LDH (U/L) & $0-1$ & $2-4$ & $5-6$ & $p$ & $\begin{array}{l}0- \\
1\end{array}$ & $\begin{array}{l}2- \\
4\end{array}$ & $\begin{array}{l}5- \\
6\end{array}$ \\
\hline RET & $199(131-372)$ & $236(113-614)$ & $244(152-426)$ & 0.010 & 20 & 42 & 51 \\
\hline TBR & $195(125-394)$ & $245(113-614)$ & $259(188-587)$ & < 0.001 & 26 & 61 & 26 \\
\hline EMP & $236(113-614)$ & $200(152-306)$ & $232(181-340)$ & 0.210 & 94 & 16 & 3 \\
\hline GGO & $224(113-397)$ & $244(153-614)$ & $261(194-587)$ & 0.049 & 74 & 21 & 18 \\
\hline CON & $233(113-614)$ & $228(131-390)$ & $293(211-426)$ & 0.115 & 88 & 19 & 6 \\
\hline NDL & $232(113-397)$ & $231(153-587)$ & $266(171-614)$ & 0.196 & 90 & 24 & 13 \\
\hline MOS & $226(113-614)$ & $267(167-354)$ & $259(220-587)$ & 0.027 & 89 & 16 & 8 \\
\hline
\end{tabular}


Table 5

Broncho-alveolar lavage biomarkers according to HRCT finding categories. Data are given as median (range). The $p$-value for statistical significance of differences $(p<0.05)$ between the groups was calculated using the Kruskal-Wallis test. Significant association are shown in bold letters and blue color for positive associations. RET = reticulation/honeycombing, TBR = traction bronchiectasis, EMP = emphysema, GGO = ground glass opacities, $\mathrm{CON}=$ consolidations, NDL = noduli, MOS = mosaic attenuation, HRCT = high-resolution computed tomography, MAK = relative BAL macrophage fraction,

$\mathrm{NEU}$ = relative $\mathrm{BAL}$ neutrophil fraction, $\mathrm{EOS}$ = relative $\mathrm{BAL}$ eosinophil fraction, $\mathrm{LYM}$ = relative $\mathrm{BAL}$ lymphocyte fraction

\begin{tabular}{|c|c|c|c|c|c|c|c|}
\hline \multicolumn{4}{|c|}{ Median in HRCT score groups (range) } & \multirow[t]{2}{*}{$\mathbf{p}$} & \multicolumn{3}{|l|}{$\mathbf{n}$} \\
\hline MAK (\%) & $0-1$ & $2-4$ & $5-6$ & & $0-1$ & $2-4$ & $5-6$ \\
\hline RET & $37.0(6.0-95.0)$ & $49.5(5.0-98.0)$ & $62.0(5.0-93.0)$ & 0.694 & 13 & 18 & 35 \\
\hline TBR & $25.0(5.0-98.0)$ & $61.0(5.0-98.0)$ & $67.0(7.0-90.0)$ & 0.327 & 17 & 35 & 14 \\
\hline EMP & $61.0(5.0-98.0)$ & $48.5(7.0-84.0)$ & $7.0(7.0-7.0)$ & 0.308 & 55 & 10 & 1 \\
\hline GGO & $57.0(5.0-98.0)$ & $52.5(5.0-82.0)$ & $34.5(7.0-87.0)$ & 0.565 & 46 & 10 & 10 \\
\hline $\mathrm{CON}$ & $53.0(5.0-98.0)$ & $46.0(6.0-90.0)$ & $69.0(19.0-87.0)$ & 0.908 & 50 & 13 & 3 \\
\hline NDL & $63.0(6.0-98.0)$ & $46.5(17.0-95.0)$ & $37.0(5.0-85.0)$ & 0.405 & 42 & 14 & 10 \\
\hline MOS & $61.5(5.0-98.0)$ & $53.0(23.0-93.0)$ & $20.0(5.0-66.0)$ & 0.092 & 54 & 7 & 5 \\
\hline NEU (\%) & $0-1$ & $2-4$ & $5-6$ & p & $0-1$ & $2-4$ & $5-6$ \\
\hline RET & $12.0(1.0-65.0)$ & $7.5(0.0-89.0)$ & $10.0(0.0-84.0)$ & 0.520 & 13 & 16 & 33 \\
\hline TBR & $11.0(0.0-89.0)$ & $7.5(0.0-84.0)$ & $13.0(1.0-64.0)$ & 0.691 & 16 & 32 & 14 \\
\hline EMP & $10.0(0.0-89.0)$ & $8.0(0.0-66.0)$ & $58.0(58.0-58.0)$ & 0.452 & 52 & 9 & 1 \\
\hline GGO & $10.0(0.0-89.0)$ & $6.5(0.0-24.0)$ & $9.5(1.0-61.0)$ & 0.209 & 42 & 10 & 10 \\
\hline CON & $9.5(0.0-89.0)$ & $10.0(2.0-61.0)$ & $7.0(3.0-19.0)$ & 0.873 & 46 & 13 & 3 \\
\hline NDL & $10.0(0.0-89.0)$ & $10.5(1.0-70.0)$ & $10.0(0.0-84.0)$ & 0.914 & 39 & 14 & 9 \\
\hline MOS & $9.0(0.0-89.0)$ & $10.0(4.0-70.0)$ & $12.0(1.0-84.0)$ & 0.739 & 50 & 7 & 5 \\
\hline EOS (\%) & $0-1$ & $2-4$ & $5-6$ & p & $0-1$ & $2-4$ & $5-6$ \\
\hline RET & $3.0(2.0-4.0)$ & $4.0(0.0-23.0)$ & $4.0(0.0-55.0)$ & 0.876 & 4 & 11 & 21 \\
\hline TBR & $3.5(0.0-4.0)$ & $3.0(0.0-55.0)$ & $5.0(0.0-19.0)$ & 0.699 & 6 & 21 & 9 \\
\hline EMP & $3.0(0.0-55.0)$ & $6.0(0.0-23.0)$ & - & 0.547 & 29 & 7 & - \\
\hline GGO & $3.0(0.0-23.0)$ & $4.0(0.0-55.0)$ & $5.0(1.0-10.0)$ & 0.891 & 26 & 5 & 5 \\
\hline $\mathrm{CON}$ & $3.5(0.0-55.0)$ & $3.0(1.0-19.0)$ & $4.0(4.0-4.0)$ & 0.978 & 28 & 7 & 1 \\
\hline NDL & $3.0(0.0-55.0)$ & $3.0(0.0-13.0)$ & $4.5(0.0-12.0)$ & 0.835 & 21 & 9 & 6 \\
\hline
\end{tabular}




\begin{tabular}{|c|c|c|c|c|c|c|c|}
\hline \multicolumn{4}{|c|}{ Median in HRCT score groups (range) } & \multirow[t]{2}{*}{$\mathbf{p}$} & \multicolumn{3}{|l|}{$\mathbf{n}$} \\
\hline MAK (\%) & $0-1$ & $2-4$ & $5-6$ & & $0-1$ & $2-4$ & $5-6$ \\
\hline MOS & $3.0(0.0-55.0)$ & $7.0(0.0-13.0)$ & $5.0(3.0-6.0)$ & 0.794 & 29 & 4 & 3 \\
\hline LYM (\%) & $0-1$ & $2-4$ & $5-6$ & $\mathbf{p}$ & $0-1$ & $2-4$ & $5-6$ \\
\hline RET & $15.0(2.0-68.0)$ & $13.0(0.0-89.0)$ & $11.0(0.0-88.0)$ & 0.578 & 12 & 17 & 34 \\
\hline TBR & $11.0(0.0-88.0)$ & $13.0(0.0-89.0)$ & $11.5(4.0-74.0)$ & 0.921 & 15 & 34 & 14 \\
\hline EMP & $14.5(1.0-89.0)$ & $7.5(0.0-43.0)$ & $4.0(4.0-4.0)$ & 0.249 & 52 & 10 & 1 \\
\hline GGO & $7.5(0.0-77.0)$ & $21.0(7.0-89.0)$ & $17.0(4.0-88.0)$ & 0.017 & 44 & 10 & 9 \\
\hline CON & $9.5(0.0-89.0)$ & $17.5(2.0-77.0)$ & $24.0(16.0-54.0)$ & 0.783 & 48 & 12 & 3 \\
\hline NDL & $9.0(0.0-88.0)$ & $16.5(2.0-74.0)$ & $23.0(2.0-89.0)$ & 0.344 & 41 & 14 & 8 \\
\hline MOS & $11.0(0.0-89.0)$ & $15.0(3.0-33.0)$ & $33.0(2.0-74.0)$ & 0.223 & 51 & 7 & 5 \\
\hline
\end{tabular}

\section{Conclusion}

In synopsis, our analyses indicate that PBL lymphocyte and eosinophil fraction, neutrophil-lymphocyte ratio (NLR), CRP as well as BAL lymphocyte fraction may have clinically relevant implications in differing HRCT abnormalities indicating either active inflammation (ground glass opacities, consolidations, noduli, mosaic attenuation) or non-inflammatory/fibrotic processes (reticulation/honeycombing, traction bronchiectasis, emphysema).

Blood lymphocyte count is of known prognostic relevance in systemic disorders like SSC or malignancy. [28-30] Our finding, that lymphocyte fraction was consistently negatively correlated to HRCT findings considered "inflammatory" suggests, that similar mechanisms may be present in ILD patients with such phenotype. In BAL however, lymphocyte fraction showed the opposite behavior with distinctly higher values in the presence of GGO and CON, but also NOD and MOS. Lymphocytosis in BAL is a common finding in inflammatory ILD presenting with patterns of NSIP or OP, $[5,31,32]$ and especially in ILD associated with formation of granulomas, such as sarcoidosis or hypersensitivity pneumonitis.[24] Conversely, BAL lymphocyte counts are reportedly lower with increasing fibrosis.[7, 33]

Similar to our observation of PBL eosinophil counts being positively correlated with RET and TBR, blood eosinophilia has been found to be associated with disease severity in SSCILD.[34] Slightly elevated eosinophil counts in BAL fluid have repeatedly been reported in IPF patients as well as in fibrotic rather than in cellular NSIP.[7, 31, 33] In our patient cohort, such relationship with BAL eosinophil fraction could not be shown, possibly due to the low number of patients presenting with significant BAL eosinophilia.

The close relationship observed between CRP and GGO/CON resembles reports of commonly elevated CRP in ILD patients presenting with patterns like NSIP or OP. $[5,7,14]$ In ILD in the context of autoimmune 
disorders, alterations to systemic inflammatory parameters like CRP have been frequently reported and also pose a risk factor for the development of pulmonary involvement in such conditions.[29, 35-37] Contrary to CRP, we found lactate dehydrogenase (LDH) significantly positively correlated with multiple non-inflammatory/fibrotic as well as inflammatory HRCT finding scores. Elevations in LDH have been reported in IPF, where they may be associated with functional impairment and have prognostic properties. [38] Our findings however suggest, that LDH may rather be a biomarker of general disease severity than of its underlying pathogenetic processes.

Our reported study has several limitations that need to be addressed: Next to its retrospective, single center approach, the sample size was limited. The reported collective represents a heterogeneous group of several different ILD entities also including a minority of patients without signs of reticulation or honeycombing (17\%). The study collective was derived from patients subsequently discussed by the local ILD-board, which could have caused the increased inclusion of rather more complex ILD, while more typical ILD like for example sarcoidosis may be underrepresented. However, our reported evaluation did explicitly not focus on distinct ILD diagnoses, but on HRCT imaging findings and their association with biomarkers of systemic and local inflammation. Radiological assessment was not done in a blinded fashion but in the presence of the ILD-board, which reflects the multidisciplinary approach to ILD. Our reported scoring system has not been validated in a larger patient cohort but is simple to perform and does not require additional tools like special software. It was not our aim to create a comprehensive HRCT quantification and classification tool but rather to allow quantitative statistical analyses beyond only "present or absent", with a semi-quantitative dimension. It is obvious that peripheral blood cell counts as well as inflammation biomarkers like CRP or LDH can be substantially altered by infections, neoplastic or hematological conditions. Also, BAL differential cell counts can be influenced by presence of infection as well as by smoking status or age.[25,39] Additionally, BAL was only performed in approximately half of the patient collective, for it had either been done previously or it was deemed clinically unnecessary. This could have led to a selection of patients with uncommon presentation in HRCT or with rather acute than chronic ILD, as suggested by the comparably high mean lymphocyte and neutrophil counts reported. Concerning statistical methods, we acknowledge, that numerous associations have been evaluated for statistical significance, which brings up the issue of multiple testing. It was not our aim to test for significance of certain associations, but rather to apply an experimental, hypothesisbuilding approach aiming to extract clinically relevant biomarkers from a large, diverse dataset. Thus, we primarily used descriptive statistical evaluation like Spearman correlation coefficients and graphical presentation. We chose clinically meaningful quantification categories (no or little, medium, or abundant involvement) for HRCT findings and used the Kruskal-Wallis test due to its robustness against outliers for significance testing.

\section{Conclusions}

We conclude that blood lymphocyte and eosinophil fraction, NLR, CRP and BAL lymphocyte fraction may help to differ between non-inflammatory/fibrotic and active inflammatory ILD phenotypes. Especially in ILD with multiple coexisting HRCT abnormalities, these biomarkers could aid the decision whether to 
primarily initiate anti-inflammatory or antifibrotic treatment. Further prospective and larger-scale trials are warranted to evaluate the implications of such biomarkers on response to either therapeutic approach.

\section{Declarations}

\section{Funding}

No external funding was used in conducting the study or in writing of this manuscript.

\section{Conflicts of interest/Competing interests}

The authors declare no conflict of interest.

\section{Ethics approval}

This study was conducted in accordance with the Declaration of Helsinki and approved by the ethics committee of the federal state of Upper-Austria (EK Nr. 1-26-17). It was conducted in an entirely retrospective fashion, without an experimental approach or additional patient contact. Only patient data assessed in clinical routine were analyzed. Data were collected in an anonymized fashion and securely electronically stored in a way, that only the authors had access to the data. No identifiable patient data has been or will ever be published by the authors.

\section{Consent to participate}

According to the ethic committee approval, no patient consent was necessary for participation in this study.

\section{Consent for publication}

Not applicable as stated above.

\section{Availability of data and material}

According to the terms imposed by the ethics committees, the full datasets analyzed during the current study cannot be made publicly available, as they contain possibly identifiable patient data. Upon reasonable request to the corresponding author and if approved as an amendment by the responsible local ethics committee, selected anonymized data can however be shared.

\section{Code availability}

Not applicable.

\section{Authors' Contributions}


All authors have approved the paper, are able to verify the validity of the results reported and meet the criteria for authorship as established by the International Committee of Medical Journal Editors.

Conceptualization, DL, KA, HP and BL; Methodology, DL, AH, BK, HP and BL; Software, BK; Validation, DL, $\mathrm{KA}, \mathrm{AH}, \mathrm{MH}$, and $\mathrm{BK}$; Formal analysis, $\mathrm{DL}$ and $\mathrm{BK}$; Investigation, $\mathrm{DL}, \mathrm{KA}, \mathrm{AH}, \mathrm{MH}$ and $\mathrm{BL}$; Resources, DL, KA and BL; Data Curation, DL, KA and BK; Writing - Original Draft Preparation, DL, KA, AH and MH; Writing Review \& Editing, DL, KA, AH, MH, BK, HP and BL; Visualization, DL, AH, BK, HP and BL; Supervision, DL and $\mathrm{BL}$; Project Administration, $\mathrm{DL}$ and $\mathrm{BL}$; Funding Acquisition, not applicable

\section{Acknowledgements}

None.

\section{References}

1. Richeldi L, Cottin V, du Bois RM, et al (2016) Nintedanib in patients with idiopathic pulmonary fibrosis: Combined evidence from the TOMORROW and INPULSIS ${ }^{\circledR}$ trials. Respir Med 113:74-79. https://doi.org/10.1016/j.rmed.2016.02.001

2. Noble PW, Albera C, Bradford WZ, et al (2016) Pirfenidone for idiopathic pulmonary fibrosis: analysis of pooled data from three multinational phase 3 trials. Eur Respir J 47:243-253. https://doi.org/10.1183/13993003.00026-2015

3. Maher TM, Corte TJ, Fischer A, et al (2019) Pirfenidone in patients with unclassifiable progressive fibrosing interstitial lung disease: a double-blind, randomised, placebo-controlled, phase 2 trial. Lancet Respir Med. https://doi.org/10.1016/S2213-2600(19)30341-8

4. Flaherty KR, Wells AU, Cottin V, et al (2019) Nintedanib in Progressive Fibrosing Interstitial Lung Diseases. N Engl J Med 381:1718-1727. https://doi.org/10.1056/NEJMoa1908681

5. Ito Y, Arita M, Kumagai S, et al (2017) Serological and morphological prognostic factors in patients with interstitial pneumonia with autoimmune features. BMC Pulm Med 17:111. https://doi.org/10.1186/s12890-017-0453-z

6. Tashkin DP, Roth MD, Clements PJ, et al (2016) Mycophenolate mofetil versus oral cyclophosphamide in scleroderma-related interstitial lung disease (SLS II): a randomised controlled, double-blind, parallel group trial. Lancet Respir Med 4:708-719. https://doi.org/10.1016/S22132600(16)30152-7

7. Park IN, Jegal Y, Kim DS, et al (2009) Clinical course and lung function change of idiopathic nonspecific interstitial pneumonia. Eur Respir J 33:68-76. https://doi.org/10.1183/09031936.00158507

8. Heukels P, Moor CC, von der Thüsen JH, et al (2019) Inflammation and immunity in IPF pathogenesis and treatment. Respir Med 147:79-91. https://doi.org/10.1016/j.rmed.2018.12.015

9. Idiopathic Pulmonary Fibrosis Clinical Research Network, Raghu G, Anstrom KJ, et al (2012) Prednisone, azathioprine, and N-acetylcysteine for pulmonary fibrosis. N Engl J Med 366:1968-77. 
https://doi.org/10.1056/NEJMoa1113354

10. Kolb $M$, Vašáková $M(2019)$ The natural history of progressive fibrosing interstitial lung diseases. Respir Res 20:57. https://doi.org/10.1186/s12931-019-1022-1

11. Khanna D, Denton CP, Lin CJF, et al (2018) Safety and efficacy of subcutaneous tocilizumab in systemic sclerosis: results from the open-label period of a phase II randomised controlled trial (faSScinate). Ann Rheum Dis 77:212-220. https://doi.org/10.1136/annrheumdis-2017-211682

12. Distler O, Highland KB, Gahlemann M, et al (2019) Nintedanib for Systemic Sclerosis-Associated Interstitial Lung Disease. N Engl J Med 380:2518-2528. https://doi.org/10.1056/NEJMoa1903076

13. Walsh SLF, Devaraj A, Enghelmayer JI, et al (2018) Role of imaging in progressive-fibrosing interstitial lung diseases. Eur Respir Rev 27:180073. https://doi.org/10.1183/16000617.0073-2018

14. Jeny F, Brillet P-Y, Kim Y-W, et al (2019) The place of high-resolution computed tomography imaging in the investigation of interstitial lung disease. Expert Rev Respir Med 13:79-94. https://doi.org/10.1080/17476348.2019.1556639

15. Salisbury ML, Tolle LB, Xia M, et al (2017) Possible UIP pattern on high-resolution computed tomography is associated with better survival than definite UIP in IPF patients. Respir Med 131:229235. https://doi.org/10.1016/j.rmed.2017.08.025

16. Flaherty KR (2003) Radiological versus histological diagnosis in UIP and NSIP: survival implications. Thorax 58:143-148. https://doi.org/10.1136/thorax.58.2.143

17. Yunt ZX, Chung JH, Hobbs S, et al (2017) High resolution computed tomography pattern of usual interstitial pneumonia in rheumatoid arthritis-associated interstitial lung disease: Relationship to survival. Respir Med 126:100-104. https://doi.org/10.1016/j.rmed.2017.03.027

18. Lynch DA, Sverzellati N, Travis WD, et al (2018) Diagnostic criteria for idiopathic pulmonary fibrosis: a Fleischner Society White Paper. Lancet Respir Med 6:138-153. https://doi.org/10.1016/S22132600(17)30433-2

19. Raghu G, Remy-Jardin M, Myers JL, et al (2018) Diagnosis of Idiopathic Pulmonary Fibrosis. An Official ATS/ERS/JRS/ALAT Clinical Practice Guideline. Am J Respir Crit Care Med 198:e44-e68. https://doi.org/10.1164/rccm.201807-1255ST

20. von Elm E, Altman DG, Egger M, et al (2007) The Strengthening the Reporting of Observational Studies in Epidemiology (STROBE) statement: guidelines for reporting observational studies. Lancet 370:1453-1457. https://doi.org/10.1016/S0140-6736(07)61602-X

21. Fischer A, Antoniou KM, Brown KK, et al (2015) An official European Respiratory Society/American Thoracic Society research statement: interstitial pneumonia with autoimmune features. Eur Respir J 46:976-987. https://doi.org/10.1183/13993003.00150-2015

22. Gruden JF, Naidich DP, Machnicki SC, et al (2019) An Algorithmic Approach to the Interpretation of Diffuse Lung Disease on Chest CT Imaging. Chest. https://doi.org/10.1016/j.chest.2019.10.017

23. Kim M, Lee SM, Song J-W, et al (2017) Added value of prone CT in the assessment of honeycombing and classification of usual interstitial pneumonia pattern. Eur J Radiol 91:66-70.

https://doi.org/10.1016/j.ejrad.2017.03.018

Page 18/22 
24. Meyer KC (2014) The clinical utility of bronchoalveolar lavage in interstitial lung disease - is it really useful? Expert Rev Respir Med 8:133-135. https://doi.org/10.1586/17476348.2014.879827

25. Meyer KC, Raghu G, Baughman RP, et al (2012) An Official American Thoracic Society Clinical Practice Guideline: The Clinical Utility of Bronchoalveolar Lavage Cellular Analysis in Interstitial Lung Disease. Am J Respir Crit Care Med 185:1004-1014. https://doi.org/10.1164/rccm.201202-0320ST

26. Lang D, Akbari K, Lamprecht B, Fellner F (2018) Initial Patient Characteristics and Standardized Quantification of Radiological Findings in Nonspecific Interstitial Pneumonia. Pneumologie 72:S48S48. https://doi.org/10.1055/s-0037-1619245

27. Lang D, Akbari K, Horner A, et al (2020) Standardized Computed Tomography Findings as Determinants of Local and Systemic Inflammation in Interstitial Lung Disease Patients. In: B42. ILD EPIDEMIOLOGY I. American Thoracic Society, pp A3392-A3392

28. Gambichler T, Tigges C, Burkert B, et al (2010) Absolute count of $T$ and B lymphocyte subsets is decreased in systemic sclerosis. Eur J Med Res 15:44. https://doi.org/10.1186/2047-783X-15-1-44

29. Bonhomme O, André B, Gester F, et al (2019) Biomarkers in systemic sclerosis-associated interstitial lung disease: review of the literature. Rheumatology 58:1534-1546. https://doi.org/10.1093/rheumatology/kez230

30. Huemer, Lang, Westphal, et al (2019) Baseline Absolute Lymphocyte Count and ECOG Performance Score Are Associated with Survival in Advanced Non-Small Cell Lung Cancer Undergoing PD-1/PD-L1 Blockade. J Clin Med 8:1014. https://doi.org/10.3390/jcm8071014

31. Welker $L$ (2004) Predictive value of BAL cell differentials in the diagnosis of interstitial lung diseases. Eur Respir J 24:1000-1006. https://doi.org/10.1183/09031936.04.00101303

32. Costabel U, Bonella F, Ohshimo S, Guzman J (2010) Diagnostic Modalities in Sarcoidosis: BAL, EBUS, and PET. Semin Respir Crit Care Med 31:404-408. https://doi.org/10.1055/s-0030-1262207

33. Veeraraghavan S, Latsi PI, Wells AU, et al (2003) BAL findings in idiopathic nonspecific interstitial pneumonia and usual interstitial pneumonia. Eur Respir J 22:239-244.

https://doi.org/10.1183/09031936.03.00105202

34. Ando K, Nakashita T, Kaneko N, et al (2016) Associations between peripheral blood eosinophil counts in patients with systemic sclerosis and disease severity. Springerplus 5:1401. https://doi.org/10.1186/s40064-016-3106-4

35. Zhang L, Wu G, Gao D, et al (2016) Factors Associated with Interstitial Lung Disease in Patients with Polymyositis and Dermatomyositis: A Systematic Review and Meta-Analysis. PLoS One 11:e0155381. https://doi.org/10.1371/journal.pone.0155381

36. Gao H, Zhang X-W, He J, et al (2018) Prevalence, risk factors, and prognosis of interstitial lung disease in a large cohort of Chinese primary Sjögren syndrome patients. Medicine (Baltimore) 97:e11003. https://doi.org/10.1097/MD.0000000000011003

37. Liu X, Mayes MD, Pedroza C, et al (2013) Does C-Reactive Protein Predict the Long-Term Progression of Interstitial Lung Disease and Survival in Patients With Early Systemic Sclerosis? Arthritis Care Res (Hoboken) 65:1375-1380. https://doi.org/10.1002/acr.21968 
38. Åttingsberg E, Hoyer N, Wilcke T, et al (2019) Lactate dehydrogenase as a biomarker of advanced disease in idiopathic pulmonary fibrosis. In: Idiopathic interstitial pneumonias. European Respiratory Society, p PA4701

39. Gharsalli H, Mlika M, Sahnoun I, et al (2018) The utility of bronchoalveolar lavage in the evaluation of interstitial lung diseases: A clinicopathological perspective. Semin Diagn Pathol 35:280-287. https://doi.org/10.1053/j.semdp.2018.08.003

\section{Figures}




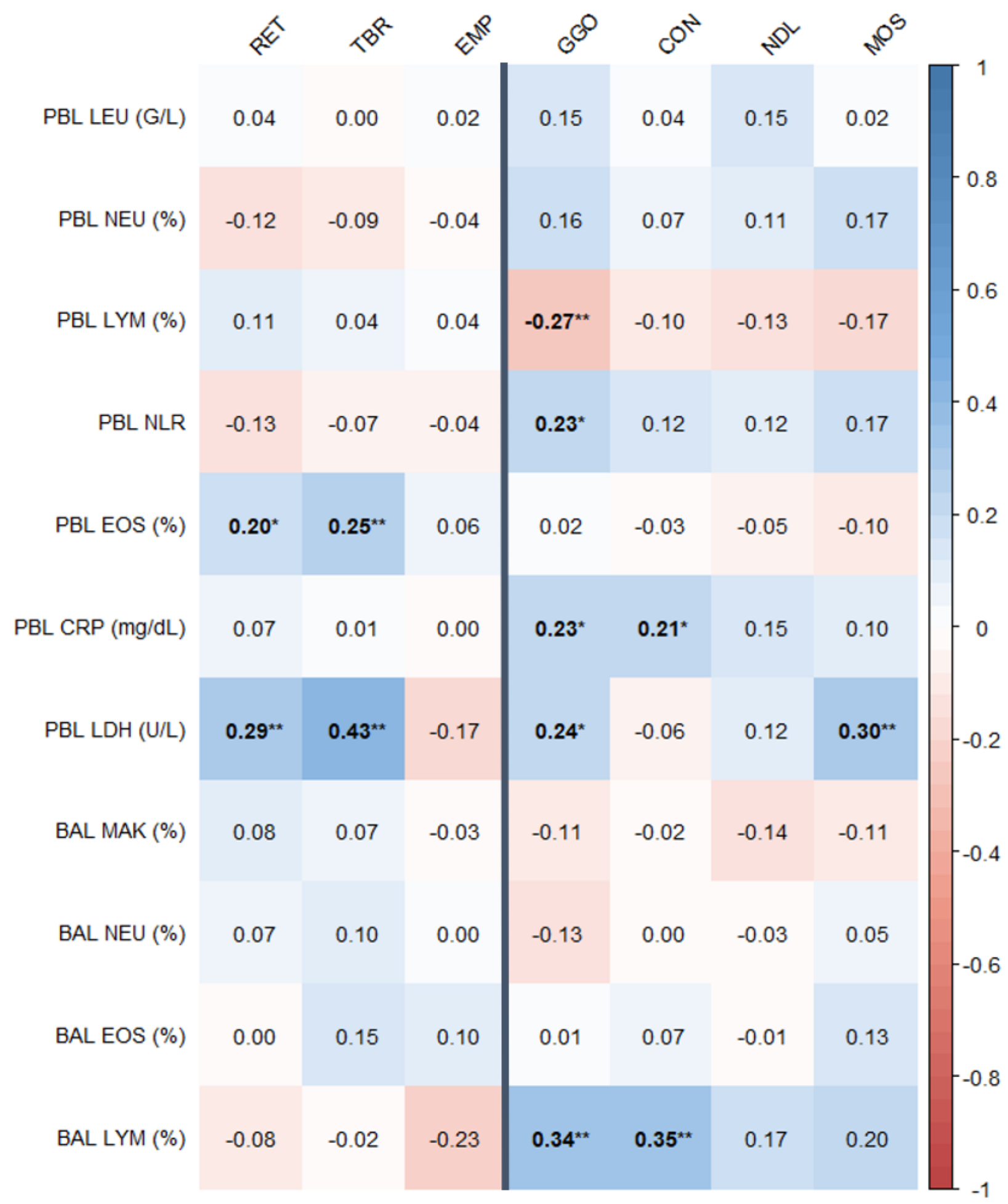

\section{Figure 1}

Correlation matrix of peripheral blood and BAL biomarkers with HRCT finding scores. Values are for Spearman correlation coefficients; colors indicate strength and direction of correlations as shown by the scale on the right side. Bold numbers are for significant correlations, $\star_{i}$ for $p<0.05, * \star_{i s}$ for $p<0.01$. The line between the EMP and GGO category visually separates non-inflammatory from inflammatory HRCT findings. $\mathrm{PBL}=$ peripheral blood, $\mathrm{BAL}=$ bronchoalveolar lavage, $\mathrm{HRCT}=$ high-resolution computed 
tomography, RET=reticulation/honeycombing, TBR=traction bronchiectasis, EMP=emphysema, $\mathrm{GGO}=$ ground glass opacities, $\mathrm{CON}=$ consolidations, $\mathrm{NDL}=$ noduli, $\mathrm{MOS}=$ mosaic attenuation, $\mathrm{LEU}=$ absolute blood leukocyte count, NEU=relative neutrophil fraction, LYM= relative lymphocyte fraction, NLR= neutrophil to lymphocyte fraction, EOS=relative eosinophil count, $\mathrm{CRP}=\mathrm{C}$-reactive protein, $\mathrm{LDH}=$ lactate dehydrogenase, MAK=relative macrophage fraction 